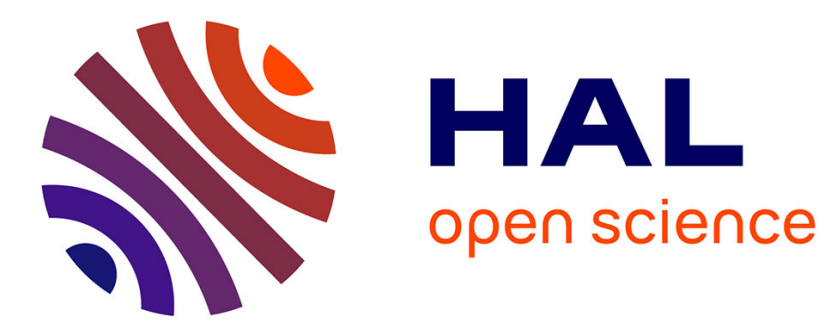

\title{
Étude des phonons optiques de grande longueur d'onde dans les alliages Zn1-xMgxTe
}

L.K. Vodop'Yanov, E.A. Vinogradov, N.N. Melnik, V.G. Plotnitchenko, Jacques Chevallier, J.C. Guillaume

\section{To cite this version:}

L.K. Vodop'Yanov, E.A. Vinogradov, N.N. Melnik, V.G. Plotnitchenko, Jacques Chevallier, et al.. Étude des phonons optiques de grande longueur d'onde dans les alliages Zn1-xMgxTe. Journal de Physique, 1978, 39 (6), pp.627-630. 10.1051/jphys:01978003906062700 . jpa-00208795

\section{HAL Id: jpa-00208795 https://hal.science/jpa-00208795}

Submitted on 1 Jan 1978

HAL is a multi-disciplinary open access archive for the deposit and dissemination of scientific research documents, whether they are published or not. The documents may come from teaching and research institutions in France or abroad, or from public or private research centers.
L'archive ouverte pluridisciplinaire HAL, est destinée au dépôt et à la diffusion de documents scientifiques de niveau recherche, publiés ou non, émanant des établissements d'enseignement et de recherche français ou étrangers, des laboratoires publics ou privés. 


\author{
Classification \\ Physics Abstracts \\ $78.30-63.20-63.20 \mathrm{D}$

\section{ÉTUDE DES PHONONS OPTIQUES DE GRANDE LONGUEUR D'ONDE DANS LES ALLIAGES $\mathrm{Zn}_{1-x} \mathrm{Mg}_{x} \mathrm{Te}$}

\author{
L. K. VODOP'YANOV, E. A. VINOGRADOV, N. N. MELNIK, V. G. PLOTNITCHENKO \\ Institut de Physique de l'Académie des Sciences (F.I.A.N.), Moscou, U.S.S.R.
}

J. CHEVAllier et J. C. GUILlaume

Laboratoire de Physique des Solides, C.N.R.S.,

1, place Aristide-Briand, 92190 Meudon Bellevue, France.

(Reçu le 21 novembre 1977, accepté le 14 février 1978)

\begin{abstract}
Résumé. - Les spectres de réflexion dans l'infrarouge dans la gamme 20-80 $\mu$ et les spectres de diffusion Raman ont été mesurés pour les alliages ternaires $\mathrm{Zn}_{1-x} \mathrm{Mg}_{x} \mathrm{Te}$ dans la gamme de compositions $0<x<0,45$. La variation énergétique des phonons optiques de grande longueur d'onde en fonction de la composition a été déduite. Ces alliages se comportent comme un système à deux modes. L'extrapolation des valeurs expérimentales à MgTe donne : $\omega_{\text {TO }}=237 \mathrm{~cm}^{-1}$ et $\omega_{\text {LO }}=305 \mathrm{~cm}^{-1}$. Aucune anomalie n'apparaît dans la variation des énergies de phonons au voisinage du point de transition des phases cristallines. Dans ces alliages, la substitution des atomes de zinc et de magnésium se fait de façon aléatoire.
\end{abstract}

Abstract. - Reflectivity spectra in the range 20-80 $\mu$ and Raman scattering spectra have been measured for $\mathrm{Zn}_{1-x} \mathrm{Mg}_{x} \mathrm{Te}$ ternary alloys in the composition range $0<x<0.45$. The energy variation of long wavelength optical phonons versus composition has been deduced. These alloys have a two mode behaviour. The extrapolation of experimental values to $\mathrm{MgTe}$ gives : $\omega_{\mathrm{TO}}=237 \mathrm{~cm}^{-1}$ and $\omega_{\mathrm{LO}}=305 \mathrm{~cm}^{-1}$. No anomaly appears in the phonon energy variation near the transition point of crystalline phases. In these alloys, the zinc and magnesium atoms are randomly substituted in the lattice.

1. Introduction. - Les spectres de phonons des alliages II-VI ont été intensément étudiés ces dernières années. Cependant, quelques problèmes de dynamique de réseau de ces cristaux demeurent non résolus comme, par exemple, l'influence d'une transition de phases sur les spectres de phonons d'un système ternaire. Un autre problème est lié à la vérification expérimentale des modèles de substitution des atomes dans un alliage ternaire : il est intéressant de vérifier si des agrégats se forment ou si cette substitution a un caractère aléatoire.

La croissance d'alliages entre $\mathrm{ZnTe}$ et $\mathrm{MgTe}$ nous a permis de réaliser des expériences dans le but de résoudre quelques-uns de ces problèmes. Un de ces composés, $\mathrm{ZnTe}$, a la structure blende de zinc alors que l'autre, MgTe a la structure wurtzite. Par ailleurs, il était intéressant d'étudier les spectres de vibrations de ces nouveaux alliages ternaires en utilisant les méthodes optiques : réflectivité infrarouge (R.I.) et diffusion Raman (D.R.).
2. Méthodes expérimentales. - Les cristaux ont été préparés par la méthode de Bridgman [1]. Dans un bain de tellure et de magnésium, on dissout des cristaux de $\mathrm{MgZn}_{2}$. Le magnésium, le zinc et le tellure utilisés ont la pureté $6 \mathrm{~N}$. Après réaction de synthèse à $600^{\circ} \mathrm{C}$, l'ampoule est portée à $1150^{\circ} \mathrm{C}$ puis est descendue dans un gradient de température. Les alliages obtenus sont polycristallins : la taille du plus grand côté des grains est de quelques millimètres.

Les spectres de R.I. des cristaux ont été mesurés en utilisant un spectromètre standard FIS-21. En raison de la petite taille des échantillons, nous avons construit un système de focalisation de la lumière à l'aide de miroirs toroïdaux. Ce système nous a permis d'obtenir une dimension du faisceau de $3 \times 8 \mathrm{~mm}^{2}$, au point de focalisation. Les spectres de réflexion ont été mesurés à la température ambiante et en lumière non polarisée. La résolution est d'environ $2 \mathrm{~cm}^{-1}$. 
Pour les spectres de diffusion Raman, nous avons utilisé des excitations lumineuses provenant des lasers suivants : type IGLA-2 et modèle 165-03 de Spectra Physics, dont la puissance totale est de $4 \mathrm{~W}$. Les spectres ont été enregistrés à l'aide du monochromateur d'un spectromètre DFS-24. Sa gamme de longueur d'onde s'étend de 400 à $850 \mathrm{~nm}$ et sa distance focale est de $822 \mathrm{~mm}$. La détection utilise une amplification à courant continu. Toutes les mesures d'effet Raman ont été faites en réflexion. Notre bâti d'expériences nous a permis d'enregistrer des spectres de D.R. pour différentes raies d'excitation lumineuse sans changer la configuration de l'expérience ni changer le point d'excitation des échantillons.

3. Résultats et discussions. - Sur les figures 1 et 2 , on a reporté les spectres de réflexion I.R. de 4 échantillons de $\mathrm{Zn}_{1-x} \mathrm{Mg}_{x}$ Te pour $0 \leqslant x \leqslant 0,42$. On voit que pour toutes les compositions $x>0$, deux bandes de réflexion apparaissent (Bandes de Reststrahlen). L'intensité de la bande haute fréquence diminue quand l'alliage s'enrichit en $\mathrm{MgTe}$ alors que celle de la bande basse fréquence augmente. La position des bandes change peu avec la composition. Ce

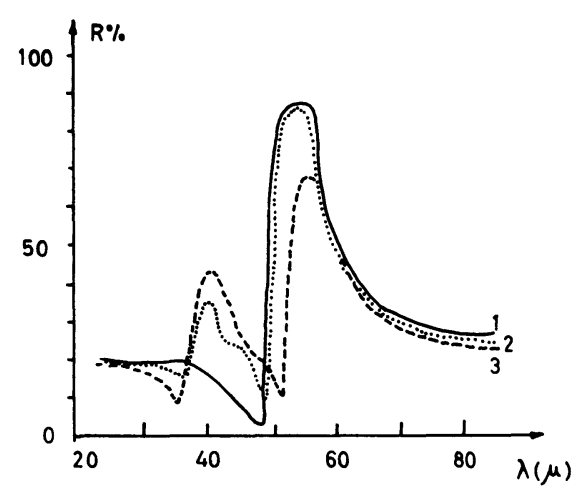

Fig. 1. - Spectres de réflectivité infrarouge d'alliages $\mathrm{Zn}_{1-x} \mathrm{Mg}_{x} \mathrm{Te}$ : $1: x=0 ; 2: x=0.1 ; 3: x=0,29$.

[Infrared reflectivity spectra of $\mathrm{Zn}_{1-x} \mathrm{Mg}_{x} \mathrm{Te}$ alloys : $1: x=0$; $2: x=0.1 ; 3: x=0.29$.]

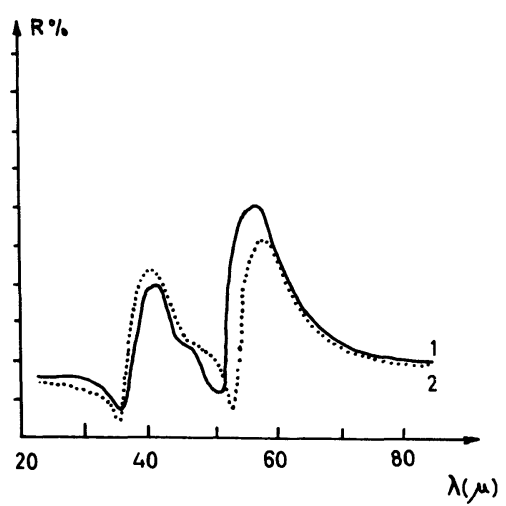

Fig. 2. - Spectres de réflectivité infrarouge pour : $1: x=0,35$; $2: x=0,42$.

[Infrared reflectivity spectra for : $1: x=0.35 ; 2: x=0.42$.] comportement nous indique que l'alliage $\mathrm{Zn}_{1-x} \mathrm{Mg}_{x} \mathrm{Te}$ a le caractère d'un système à deux modes. Les bandes haute fréquence et basse fréquence sont caractéristiques de $\mathrm{MgTe}$ et de $\mathrm{ZnTe}$ respectivement. D'autre part, l'absence de structure fine dans les spectres de R.I. apporte la preuve que la substitution des atomes de magnésium et de zinc est aléatoire [2].

Les fréquences des phonons de grande longueur d'onde ont été obtenues graphiquement en s'appuyant sur la remarque suivante : en analysant les courbes de réflexion (R.I.) de nombreux matériaux dont les fréquences de phonons au centre de la zone de Brillouin étaient connues, on a noté que les fréquences de ces phonons correspondaient aux maxima de la fonction $\mathrm{d} R(\omega) / \mathrm{d} \omega$, c'est-à-dire avec points d'inflexion des fronts de montée et de descente des bandes de Reststrahlen. Cette dépendance obtenue de façon empirique a aussi été prouvée théoriquement [3].

La variation en fréquence des phonons du système $\mathrm{Zn}_{1-x} \mathrm{Mg}_{x} \mathrm{Te}$ est présentée sur la figure 4 .

Les spectres des phonons du centre de zone ont été obtenus, pour les mêmes cristaux, en utilisant la méthode de diffusion Raman. Cette méthode permet de déterminer directement les fréquences des phonons optiques et sa précision est bien meilleure que celle de la méthode R.I. Les spectres D.R. des cristaux $\mathrm{Zn}_{1-x} \mathrm{Mg}_{x} \mathrm{Te}$ pour $x=0,37$ ont été mesurés pour différentes raies d'excitation fournies par un laser à argon ionisé (Fig. 3). Ces spectres ont une

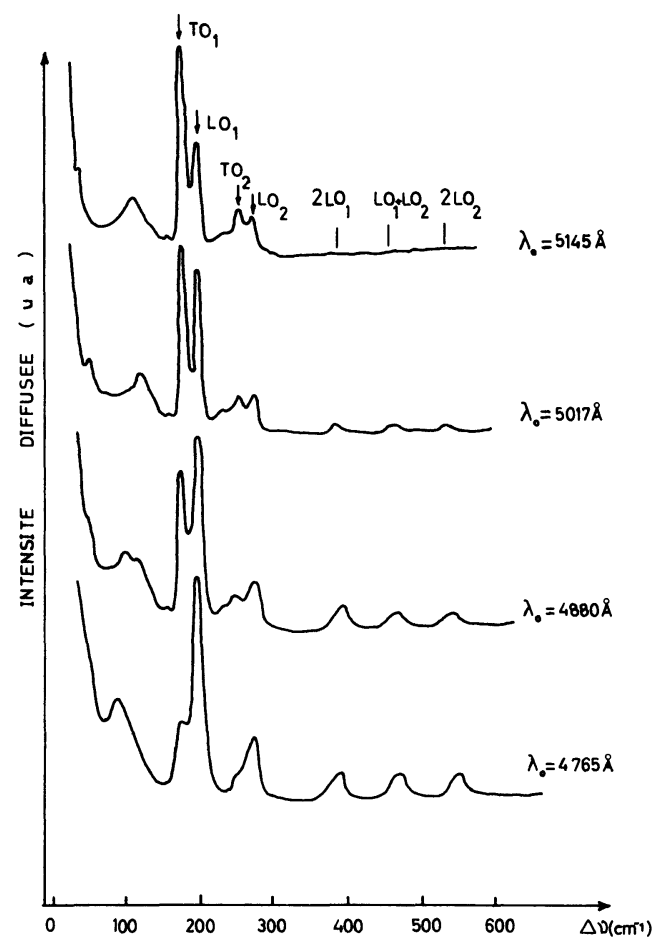

Fig. 3. - Spectres de diffusion Raman d'un cristal $\mathrm{Zn}_{0,63} \mathrm{Mg}_{0,37} \mathrm{Te}$ enregistrés à $300 \mathrm{~K}$ lors de l'excitation par quatre raies différentes d'un laser à argon ionisé. Résolution $s=2 \mathrm{~cm}^{-1}$.

[Raman scattering spectra of a crystal $\mathrm{Zn}_{0.63} \mathrm{Mg}_{0.37} \mathrm{Te}$ recorded at $300 \mathrm{~K}$ under $\mathrm{Ar}^{+}$laser excitation at four different wavelengths. Resolution $s=2 \mathrm{~cm}^{-1}$.] 
structure complexe. Afin de séparer les raies de D.R. du $1^{\text {er }}$ ordre des raies d'ordre plus élevé, on a mesuré les dépendances en température des pics D.R. dans la gamme $80 \mathrm{~K}-300 \mathrm{~K}$. Ces mesures ont montré que les raies repérées par une flèche sur la figure étaient liées aux processus D.R. du $1^{\mathrm{er}}$ ordre. D'autre part, des mesures de D.R. utilisant une lumière polarisée $\left({ }^{1}\right)$ nous ont permis de déterminer le caractère de polarisation (LO ou TO) des raies D.R. du premier ordre pour les alliages de composition riche en ZnTe. Dans ces analyses, nous avons supposé que tous les alliages de cette gamme de composition possédaient une structure blende de zinc. Nous ne sommes pas sûrs cependant de la validité de cette hypothèse pour $x \simeq 0,40$, puisque nous sommes alors dans la région où l'alliage change de structure cristallographique. Il est possible que pour ces compositions, il y ait existence de polytypes.

L'analyse des spectres D.R. du premier ordre (Tableau I) montre l'existence de deux groupes de

TABLEAU I

\begin{tabular}{lcccc}
\multicolumn{1}{c}{$x$} & $\begin{array}{c}\omega_{\mathrm{TO}_{1}} \\
\left(\mathrm{~cm}^{-1}\right)\end{array}$ & $\begin{array}{c}\omega_{\mathrm{LO}_{1}} \\
\left(\mathrm{~cm}^{-1}\right)\end{array}$ & $\begin{array}{c}\omega_{\mathrm{TO}_{2}} \\
\left(\mathrm{~cm}^{-1}\right)\end{array}$ & $\begin{array}{c}\omega_{\mathrm{LO}_{2}} \\
\left(\mathrm{~cm}^{-1}\right)\end{array}$ \\
0 & - & - & - & - \\
0,1 & 177 & 205 & - & - \\
0,29 & 178 & 199 & 245 & 265 \\
0,35 & 171 & 191 & 235 & 270 \\
0,42 & 167 & 189 & 233 & 272 \\
0,44 & 166 & 185 & 234 & 274 \\
& & & & 274 \\
0 & 177 & 205 & - & - \\
0,1 & 174 & 202 & 255 & 267 \\
0,29 & 171 & 193 & 249 & 278 \\
0,35 & 173 & 194 & 252 & 276 \\
0,37 & 168 & 188 & 245 & 279 \\
0,38 & 169 & 189 & 251 & 279 \\
0,42 & 169 & 188 & 248 & 280 \\
0,44 & 169 & 186 & 247 & 280
\end{tabular}

Réflexion Infrarouge

Diffusion Raman

raies pour les cristaux de composition $x>0$. Un des groupes $\left(\mathrm{TO}_{1}\right.$ et $\left.\mathrm{LO}_{1}\right)$ est caractéristique du réseau de $\mathrm{ZnTe}$ alors que l'autre $\left(\mathrm{TO}_{2}\right.$ et $\left.\mathrm{LO}_{2}\right)$ est caractéristique du réseau de $\mathrm{MgTe}$. Lorsque $x$ augmente, la fréquence des phonons $\mathrm{TO}_{2}, \mathrm{TO}_{1}$ et $\mathrm{LO}_{1}$ diminue alors que celle de $\mathrm{LO}_{2}$ augmente. Ce comportement est en accord avec l'évolution des spectres de phonons dans les alliages $A_{1-x} \mathrm{~B}_{x} \mathrm{C}$ du type 2 modes. La dépendance énergétique de ces phonons en fonction de la composition à partir des méthodes R.I. et D.R. est présentée sur la figure 4. Lorsque l'alliage s'enrichit en magnésium, les énergies des phonons longitudinaux et transversaux convergent vers les fréquences du mode de gap ou du mode de bande du zinc dans MgTe (la densité d'états de phonons pour MgTe n'est pas connue). Nos résultats sont en accord avec ceux obtenus tout récemment par Zigone [4].

( $\left.{ }^{1}\right)$ Pour ces mesures, nous avons découpé, dans nos polycristaux, des échantillons monocristallins orientés de dimensions

$$
1 \times 1 \times 1 \mathrm{~mm}^{3} \text {. }
$$

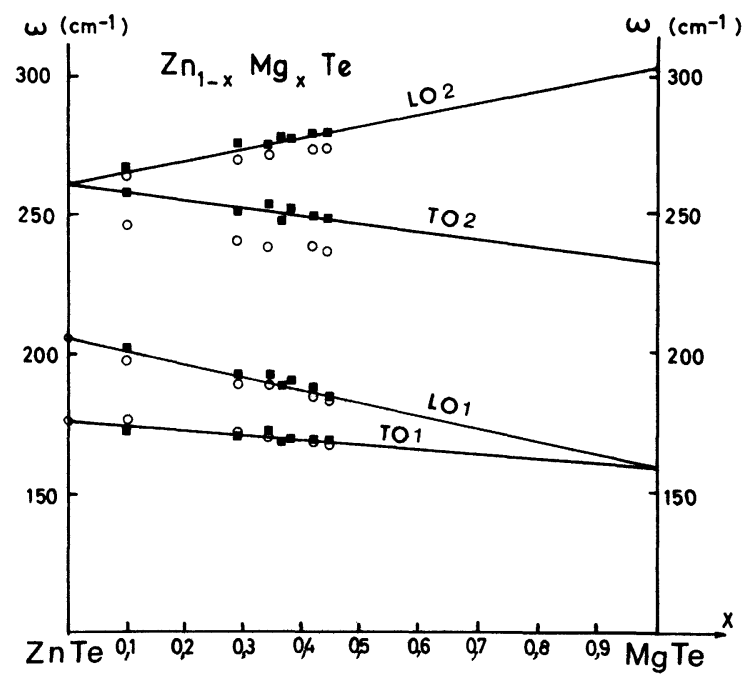

FIG. 4. - Variation de la fréquence des phonons optiques avec la composition dans les solutions solides $\mathrm{Zn}_{1-x} \mathrm{Mg}_{x} \mathrm{Te}: \mathrm{O}=$ réflectivité infrarouge $\boldsymbol{\|}=$ Diffusion Raman.

[Variation of optical phonon frequencies versus composition in $\mathrm{Zn}_{1-x} \mathrm{Mg}_{x} \mathrm{Te}$ solid solution : $\mathrm{O}=$ infrared reflectivity $\mathbf{~ R a m a n}$ scattering.]

Par extrapolation de nos valeurs expérimentales, nous avons pu estimer les fréquences des phonons TO et LO pour le composé MgTe pur : $\omega_{\mathrm{TO}}=237 \mathrm{~cm}^{-1}$ et $\omega_{\mathrm{LO}}=305 \mathrm{~cm}^{-1}$. Ces résultats sont intéressants en raison de la difficulté de réaliser des mesures directes sur MgTe qui présente une très forte réactivité avec la vapeur d'eau.

Nos valeurs extrapolées à MgTe sont en bon accord avec celles estimées par Nakashima à partir des alliages $\mathrm{Mg}_{x} \mathrm{Cd}_{1-x} \mathrm{Te}$ [5] et avec celles mesurées par Zigone pour MgTe [4].

La variation des fréquences de phonons avec la composition du système $\mathrm{Zn}_{1-x} \mathrm{Mg}_{x}$ Te est très bien décrite dans le cadre d'un modèle M.R.E.I. [6], dans lequel on suppose que les atomes qui se substituent sont distribués de façon aléatoire et que les deux sous-réseaux de cations et d'anions vibrent rigidement.

La figure 4 montre que si la composition $x$ s'approche de la zone où les 2 phases, blende de zinc et wurtzite, coexistent $(x \simeq 0,4)$, aucune particularité n'apparaît dans le comportement des spectres de phonons en fonction de la composition. Ces résultats sont en accord avec les résultats obtenus sur le système $\mathrm{CdTe}_{1-x} \mathrm{Se}_{x}$ [7].

Sur la figure 3 , nous avons reporté les spectres de D.R. pour différentes longueurs d'onde de raies excitatrices : on observe une forte variation du rapport d'intensités entre les raies du $1^{\mathrm{er}}$ et du $2^{\mathrm{e}}$ ordre. Avec une excitation de longueur d'onde $\lambda=5017 \AA$, les raies de diffusion impliquant 2 phonons $\mathrm{LO}_{1}$, 2 phonons $\mathrm{LO}_{2}$ et la combinaison $\mathrm{LO}_{1}+\mathrm{LO}_{2}$ commencent à apparaître. Les intensités de ces doubles phonons augmentent lorsque la longueur d'onde d'excitation diminue. Nous avons expliqué 
ce comportement sur la base de la diffusion Raman résonnant. Cet effet consiste en l'augmentation des raies D.R. quand l'énergie des photons de l'excitation s'approche de la valeur de la bande interdite, mais une analyse plus poussée de ces effets exige des mesures utilisant une lumière polarisée.

4. Conclusions. - Nos mesures ont montré que les alliages $\mathrm{Zn}_{x} \mathrm{Mg}_{1-x} \mathrm{Te}$ se comportent comme un système du type deux modes. La substitution des atomes de zinc et de magnésium s'y fait de façon aléatoire. Aucune anomalie n'a été observée dans la gamme de compositions où se situe le point de transition des phases. Il serait cependant intéressant d'étendre l'étude à $x>0,50$ pour confirmer ce résultat.

Remerciements. - Les auteurs remercient vivement M. Revel (C.N.R.S., Vitry) pour la fourniture de magnésium de haute pureté et $\mathrm{M}$. Rommeluere pour la croissance des cristaux d'alliages.

\section{Bibliographie}

[1] Guillaume, J. C., Chevallier, J., Rommeluere, J. F., Rouy, G., Revel, G., Rev. Phys. Appl. 11 (1976) 725.

[2] Verleur, H. et Barker, A., Phys. Rev. 149 (1966) 715.

[3] Vinogradov, E. A. et Vodop'yanov, L. K., Krat. Soobsch. Fiz. 11 (1972) 29.

[4] Zigone, M., Hirlimann, C., Jouanne, M., Chevallier, J., Martin, M. S., Proc. Int. Conf. on lattice dynamics (Paris) (1977) à paraître.
[5] Nakashima, S., Fukumoto, T., Mitsuishi, A. et Itoh, K. J. Phys. Soc. Jpn. 35 (1973) 1437.

[6] Chang, I. et Mitra, S., Adv. Phys. 20 (1971) 359.

[7] Vinogradov, E. A., Vodop'yanov, L. K. et Oleinik, G. S., Sov. Phys. Solid State 15 (1973) 322.

[8] Vodop'yanov, L. K., Vinogradov, E. A., Blinov, A. M. et Rukavishnikov, V. A., Sov. Phys. Solid State 14 (1972) 219. 